\title{
FATORES IMPACTANTES NO DESEMPENHO ORGANIZACIONAL: PROPOSIÇÃO DE UM MODELO CONCEITUAL
} IMPACT FACTORS ON ORGANIZATIONAL PERFORMANCE: PROPOSITION OF A CONCEPTUAL MODEL

DEISE TAIANA DE ÁVILA DIAS ${ }^{1}$

DEONIR DE TONI ${ }^{2}$

RESUMO: A busca por um melhor desempenho organizacional é uma prática que intriga muitos pesquisadores e gestores das organizações. A questão central de pesquisa é: quais são os construtos e dimensões que estão relacionados ao desempenho organizacional? Assim, o objetivo deste estudo consiste em identificar as dimensões e os construtos que podem explicar melhor o desempenho organizacional. A base teórica desta pesquisa foi composta pela Teoria Baseada em Recursos e pela Teoria das Capacidades Dinâmicas. O construto desempenho organizacional é composto pelas dimensões: financeira, desempenho de mercado e retorno aos acionistas. O construto gestão dos recursos é composto pelas dimensões: recursos operados e recursos operantes. O construto orientação empreendedora é composto pelas dimensões: inovatividade, assunção de riscos, proatividade, autonomia, agressividade competitiva e rede de relações. O construto de capacidades dinâmicas é composto pelas dimensões: capacidade gerencial, capacidade mercadológica e capacidade ambidestra. Dentre as contribuições deste estudo destaca-se primeiro, a evolução da compreensão dos construtos que influenciam no desempenho organizacional, segundo, pretende-se agregar mais direcionadores que impactam no desempenho organizacional, com o intuito de incentivar as práticas de gestão dos recursos e capacidades nas organizações.

Palavras-chave: Gestão dos recursos. Orientação empreendedora. Capacidades dinâmicas. Desempenho organizacional.

ABSTRACT: The search for better organizational performance is a practice that intrigues many researchers and managers of organizations. The central question of research is: what are the constructs and dimensions that are related to organizational performance? Thus, the objective of this study is to identify the dimensions and constructs that can better explain organizational performance. The theoretical basis of this research was composed by Resource Based Theory and Dynamic Capability Theory. The organizational performance construct is composed of the following dimensions: financial, market performance and return to shareholders. The resource management construct is composed of the dimensions: operated and operating resources. The entrepreneurial orientation construct is composed of the following dimensions: innovation, risk assumption, proactivity, autonomy, competitive aggressiveness and network of relationships. The dynamic capabilities construct consists of the following dimensions: managerial capacity, market capacity and ambidextrous capacity.

Data de submissão: 15/09/2017. Data de aceite: 13/12/2017. Data de publicação: 17/12/2017.

\footnotetext{
${ }^{1}$ Bacharela em Administração pela Faculdade da Serra Gaúcha, mestra em Administração pela Universidade de Caxias do Sul e doutoranda em Administração pela Universidade de Caxias do Sul. Rua Sepé Tiaraju, 161, Apto. 552, Bairro Esplanada. Caxias do Sul/RS, CEP 95095-540. e-mail: deiset.dias@gmail.com

2 Pós-Doutorado em Administração pela Universidade de São Paulo, Doutorado em Administração pela Universidade Federal do Rio Grande do Sul, Mestrado em Administração pela Universidade Federal do Rio Grande do Sul. Doutor adjunto Il da Universidade de Caxias do Sul.
} 
Among the contributions of this study, we highlight first the evolution of the understanding of the constructs that influence organizational performance; second, we intend to add more drivers that impact on organizational performance, in order to encourage the management practices of resources and capacities in the organizations.

Keywords: Resource management. Entrepreneurial orientation. Dynamic capabilities. Organizational performance.

\section{INTRODUÇÃO}

A Teoria Baseada em Recursos é uma estrutura estratégica que fornece um entendimento de por que algumas empresas superam outras. A sua importância reflete-se na sua ampla inclusão nos fatores estratégicos como uma ferramenta para avaliar os pontos fortes e fracos de uma empresa (SHEEHAN, 2006). A Teoria das Capacidades Dinâmicas surgiu reconhecendo a função da exploração do conjunto de recursos e capacidades organizacionais existentes, bem como a função da exploração de novos (combinação, integração, renovação). Por conseguinte, o pensamento das CDs toma como base a visão "schumpeteriana" evolutiva da concorrência entre as empresas. Assim, as diferenças entre as empresas originam-se por meio de novas combinações de recursos e capacidades, desenvolvidos pelas empresas ao longo de sua trajetória (TEECE; PISANO; SHUEN, 1997).

As organizações buscam combinar seus recursos tangíveis e intangíveis e competências, a fim de elaborar uma visão estratégica sistemática e dinâmica, capaz de melhorar continuamente o desempenho organizacional (MARTINHO; GOMES; YASIN, 2016). O desempenho organizacional inclui melhor aproveitamento de ativos, uma posição competitiva e lucros (ARORA; ARORA; SIVAKUMAR, 2016). O desempenho organizacional pode ser medido pelo desempenho financeiro da empresa (GREEN; INMAN, 2005), que é operacionalizado em termos de participação de mercado, retorno sobre ativos totais e crescimento de vendas (GREEN; INMAN, 2005). Assim, traz a seguinte questão de pesquisa: quais são os construtos e dimensões que estão relacionados ao desempenho organizacional?

Scott (1992) ressalta sobre a necessidade de desvendar o impacto dos diversos fatores que afetam o desempenho da empresa. Sendo assim, o objetivo deste estudo é identificar as dimensões e os construtos que podem explicar melhor o desempenho organizacional. Logo, optou-se por abordar o construto desempenho organizacional sob a ótica financeira e não financeira (GARENGO; BIAZZO; BITITCI, 2005). O desempenho organizacional é uma medida da mudança do estado financeiro de uma empresa ou dos resultados financeiros resultantes das decisões dos empreendedores e da execução dessas decisões pelos membros da organização. Uma vez que, a percepção desses resultados é contextual, as medidas utilizadas para representar o desempenho são escolhidas com base nas circunstâncias da organização, que estão sendo observadas. As medidas selecionadas representam os resultados alcançados, positivos ou negativos, sob a ótica financeira e não financeira, desempenho de mercado; e retorno aos acionistas (LI et al., 2005; CARTON; HOFER, 2006).

Este estudo está estruturado em três capítulos. O Capítulo 1 aborda a introdução, a qual apresenta, a delimitação do problema de pesquisa, o objetivo geral e o escopo da pesquisa (justificativa e relevância do estudo). O Capítulo 2 aborda o referencial teórico, compreendendo as definições dos conceitos e as discussões relacionadas aos objetivos da investigação, por meio de revisão da literatura condizente aos construtos anteriormente citados, bem como o modelo teórico proposto e as hipóteses de pesquisa. Por fim, o Capítulo 3 apresenta as considerações finais e as contribuições do estudo. 


\section{REFERENCIAL TEÓRICO}

\subsection{Desempenho organizacional}

O desempenho organizacional pode ser definido como um conjunto de resultados que a empresa vai efetivando a medida em que suas atividades são executadas, inclusive, é visto como essencial para alcançar os objetivos e metas. Assim, o sucesso da visão empresarial, pode ser entendido como um dos maiores objetivos da organização (HAKONSSON, 2006). As organizações implementam técnicas de gestão tendo como objetivo principal o seu desempenho e, consequentemente os seus resultados (ANDREWS; BOYNE, 2010; BOYNE et al., 2011). Sendo assim, o desafio das novas técnicas e abordagens na administração é promover a relação de sua implantação, juntamente com a melhoria do desempenho e do resultado organizacional. A interação entre orientação de mercado e estrutura organizacional afeta positivamente o comportamento do intraempreendedorismo, o que, por sua vez, leva ao desempenho organizacional (SUN; PAN, 2011).

A partir de seus estudos, Martins (1999) apresenta as principais características de um modelo de avaliação de desempenho, a saber: ser congruente com a estratégia competitiva; instigar e suportar a melhoria contínua; apontar tendências e progressos; propiciar o entendimento das relações de causa e efeito; ser inteligível para os membros da organização; abranger o processo em sua totalidade, desde o fornecedor até o cliente; ser dinâmico; oportunizar as informações para toda a empresa; intervir na atitude dos colaboradores; avaliar as equipes, e não indivíduos; e por fim ter medidas financeiras e não-financeiras. Assim, optou-se por abordar o desempenho mediante três fatores: (i) financeiro; (ii) desempenho de mercado; e (iii) retorno aos acionistas, conforme indicado por Engelen et al. (2015).

Em acréscimo, Adams, Khoja e Kauffman (2012) observam o desempenho organizacional como a competitividade relativa da organização em comparação com outras empresas e a capacidade de manter em longo prazo a sua rentabilidade e participação no mercado. Onde estão inclusos os fatores internos e fatores externos. Observando o posicionamento dos autores citados, neste estudo optou-se por abordar o desempenho organizacional financeiro (medidas objetivas) e não financeiro (medidas não objetivas), isto é, ele está sendo observado mediante critérios mistos (JARVIS et al., 2000; GARENGO; BIAZZO; BITITCI, 2005; SOUSA; ASPINWALL; RODRIGUES, 2006; GARENGO; NUDURUPATI; BITITCI, 2007). O desempenho organizacional investigado foi sob o ponto de vista financeiro (lucro e faturamento), mercadológico (participação de mercado e número de novos clientes) e o organizacional (retorno aos acionistas e aumento do número de colaboradores contratados).

\subsection{Gestão dos recursos, orientação empreendedora e capacidades dinâmicas}

Os recursos podem ser classificados em três principais categorias, segundo Barney (1986) e Grant (1991), a saber: (i) os recursos físicos, que incluem instalações e equipamentos, recursos naturais e matérias-primas, os quais neste estudo são chamados de recursos operados ou tangíveis; (ii) os recursos humanos, que incluem os trabalhadores produtivos, técnicos e gerenciais; e por fim (iii) os recursos organizacionais são formados pelas rotinas que coordenam os recursos humanos e físicos de forma produtiva, assim, os recursos humanos e organizacionais neste estudo são chamados de recursos operantes ou intangíveis. Alicerçados na perspectiva de que a gestão dos recursos, como pressupõe a Teoria Baseada em Recursos, é preditora do desempenho organizacional. Cabe destacar que, a caracterização de recursos utilizadas por esta pesquisa foi o proposto por Barney (1986) e Grant (1991).

A orientação empreendedora (OE) foi interpretada como uma qualidade individual do 
empreendedor, como sendo um indivíduo que constantemente desafia a incerteza do ambiente por meio da implementação de ações corporativas (MILLER; FRIESEN, 1982). O construto OE foi desenvolvido por Miller (1983), o autor refere-se a OE como o comportamento empreendedor, que reflete na forma de agir inovadora, proativa e de assumir riscos. Por conseguinte, a OE é estudada por Lumpkin e Dess (1996), onde os autores entendem que para melhor compreensão do empreendedorismo e sua relação com o desempenho organizacional, é indicado a inclusão de mais duas dimensões, que são: autonomia e agressividade competitiva. Posteriormente, além das três dimensões propostas por Miller (1983) e das duas dimensões propostas por Lumpkin e Dess (1996), os autores Mello e Leão (2005) percebem a necessidade da inserção de mais uma dimensão, que é a rede de relações.

A abordagem de capacidades dinâmicas vai além das especificidades das capacidades da organização como fonte de alcançar vantagem competitiva. Assim, uma capacidade dinâmica é "um padrão aprendido e estável de atividade coletiva por meio da qual a organização sistematicamente gera e modifica suas rotinas operacionais buscando melhorar sua efetividade". A estrutura das capacidades dinâmicas considera o empreendedor com o principal ator em uma organização, sendo responsável por reconhecer a necessidade de mudanças nos ambientes de negócios, onde há uma profunda incerteza (DRIESCH et al., 2015; TEECE, 2016). Sendo assim, para as capacidades dinâmicas do empreendedor, tratarão da sua: capacidade gerencial; capacidade mercadológica e capacidade ambidestra, alicerçadas na Teoria das Capacidades Dinâmicas.

\subsection{Construto gestão dos recursos e suas dimensões, os recursos operados e operantes}

A partir da revisão da literatura sobre os recursos operados e operantes identificou-se que os recursos físicos ou operados são aqueles que incluem itens como a infra-estrutura e os equipamentos, a localização geográfica e o acesso à matéria-prima. Estes recursos não costumam ser destacados como recursos estratégicos, visto que normalmente são de fácil acesso entre a concorrência. Todavia, para produção eles passam a ter uma função essencial no processo produtivo. Os recursos físicos, como equipamentos modernos e acesso às fontes de abastecimento facilitam a eficiência dos processos e a eficácia dos produtos, alcançando um desempenho organizacional superior (WEILL; VITALE, 2002; MORGAN et al., 2004).

O sistema de trabalho de alto desempenho é um conjunto de práticas de gestão dos recursos humanos e organizacionais, que compreende uma série de programas, processos e técnicas que são estabelecidos e aplicados de acordo com os objetivos estratégicos da organização. O sistema aumenta o conhecimento, habilidades, capacita os membros da organização a contribuir e aumenta a motivação e os esforços dos colaboradores, conduzindo a resultados organizacionais favoráveis (LU et al., 2015; OGUNYOMI; BRUNING, 2016).

Bontis (1998, p. 66) chama atenção sobre a importância do capital estrutural da organização, para que assim o colaborador possa contribuir com o desenvolvimento da empresa, e o valor é criado dentro da organização (LIN et al., 2016). O comprometimento dos indivíduos é maior quando eles são considerados como um recurso valioso para a empresa, em vez de um ativo a ser comprado e vendido (KATOU, 2012). Para melhorar o desempenho organizacional, os empreendedores e tomadores de decisão devem tornar seus sistemas de gestão de recursos humanos mais visíveis, compreensíveis, legítimos e relevantes (KATOU, 2015). Assim, é possível observar que os recursos operantes impactam positivamente no desempenho organizacional (COLLINS; CLARK, 2003). 
Os recursos operantes, a criatividade e o empreendedorismo podem, por algumas vezes, compensar as restrições impostas pelos recursos físicos (GRANDE; MADSEN; BORCH, 2011). Os recursos operantes são empregados para agir sobre os recursos operados (e outros recursos operantes, caso seja necessário). Arcand et al. (2004), Felício, Couto e Caiado (2014) e Srivastava e Dhar (2016), afirmam que os recursos operantes influenciam sobre a performance da empresa. Logo, as empresas devem facilitar os processos de integração de recursos operados e operantes, a fim de permitir um melhor nível de produto e/ou serviço e proporcionar experiências valiosas a seus clientes (LAUD et al., 2015).

Estes processos de integração de recursos são vistos como estruturas dinâmicas de intercâmbio que estão relacionados às interações entre pessoas, organizações e tecnologias (LAUD et al., 2015). Diante do cenário de constantes mutações, as empresas buscam novas e distintas formas para geração de valor, buscam uma melhor interação entre ativos tangíveis e intangíveis. Segundo Reilly e Schweihs (1998), Boulton, Libert e Samek (2001) e Allee (2008), apesar da relevante importância dos ativos intangíveis, isso não sinaliza que os tangíveis estejam perdendo sua relevância, sendo que é difícil dissociar um ativo tangível de um intangível.

Com o propósito de mostrar as relações entre os construtos, elaborou-se o modelo teórico conforme apresentado na Figura 1, bem como as respectivas hipóteses de pesquisa.

Figura 1: Modelo teórico proposto e as hipóteses de pesquisa

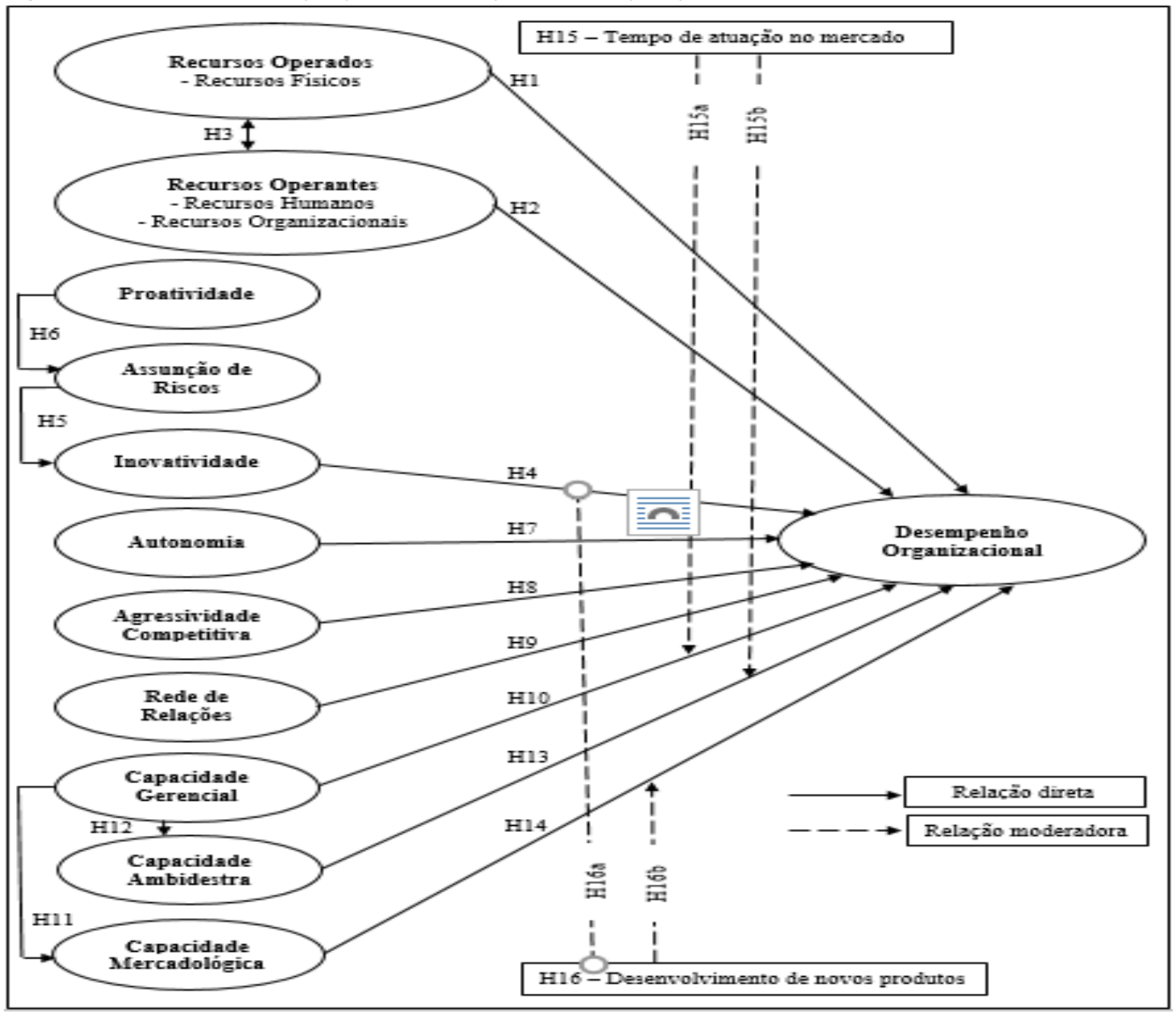

Fonte: Elaborada pelos autores. 
Cabe ressaltar que, para o desenvolvimento deste modelo teórico e as hipóteses de pesquisa, foram realizadas investigações em cinco bases de dados, que são: $E B S C O$, Emerald, $S A G E$, Scopus e Web of Science, a fim de identificar quais são os fatores que levam as empresas ao seu desempenho organizacional.

Assim, apresenta-se as seguintes hipóteses de pesquisa:

H1: Os recursos operados têm um impacto positivo e significativo no desempenho organizacional.

H2: Os recursos operantes têm um impacto positivo e significativo no desempenho organizacional.

H3: Há uma correlação positiva e significativa entre os recursos operados e operantes.

Portanto, é por meio da interação destes dois tipos de ativos (tangível e intangível) que se define o efetivo valor da organização, isto é, há uma correlação positiva entre os recursos operados e operantes. Boulton, Libert e Samek (2001) demonstraram em seus estudos que as empresas estão criando valor mediante novas formas, isso faz com que utilizem a combinação de ativos intangíveis e tangíveis, no entanto, segundo os autores, ainda não foram adequadamente reconhecidas pelos sistemas contábeis. Logo, observa-se que os recursos operados impactam nos recursos operantes, e vice-versa.

\subsection{Construto orientação empreendedora e suas dimensões, a inovatividade, assunção de riscos, proatividade, autonomia, agressividade competitiva e rede de relações}

Deste modo, a partir da revisão da literatura identificou-se que, Hult, Hurley e Knight (2004) e Veidal e Korneliussen (2013) afirmam que parte da capacidade de inovatividade da organização depende da medida em que os empreendedores adquirem e tomam suas decisões com inteligência de mercado. Mello e Leão (2005) complementam este ponto de vista que a inovatividade tem sido utilizada no desenvolvimento de práticas empreendedoras, o que impacta diretamente no desempenho organizacional (ALEGRE; CHIVA, 2013).

A literatura refere-se ao empreendedor como alguém que está disposto a assumir riscos, já que cria seu próprio negócio, incluindo seu risco pessoal. Direcionando esse pensamento para organização, as que têm uma $O E$ são caracterizadas com um comportamento de assumir riscos, como assumir amplos compromissos financeiros objetivando alcançar altos retornos por aproveitar as oportunidades no mercado, inovando com seus processos, produtos e/ou serviços (FERNÁNDEZ-MESA; ALEGRE-VIDAL; CHIVAGÓMEZ, 2012). Isto é, a assunção de riscos impacta positivamente na inovatividade, uma vez que o empreendedor que está disposto a assumir riscos aproveita novas oportunidades e está propenso a inovar nos seus processos, produtos e/ou serviços (LUMPKIN; DESS, 1996; HU, 2013; PARVEEN; JAAFAR; AININ, 2016).

Também, identificou-se que Sandberg (2002) refere-se a proatividade como um comportamento estratégico. A proatividade está relacionada a uma ação antecipada, agir antecipadamente às mudanças no ambiente, antes que tenha um impacto direto na empresa (CHEN, LI; EVANS, 2012). Desta forma, a proatividade do empreendedor tem influência na assunção de riscos (DADA; WATSON, 2013; GUO; TANG; SU, 2014).

A autonomia está atrelada a liberdade para implementar o que for necessário, a fim de antecipar e agir sobre uma oportunidade, adaptabilidade e o conhecimento aos possíveis erros (MORRIS et al., 2006) seja para o desenvolvimento de novos produtos e/ou serviços no mercado, ou mesmo empregar novas técnicas, processos e tecnologia (COVIN; MILES, 1999). Com base no posicionamento dos autores, observa-se que o intraempreendedor tem liberdade para agir, ele pode traçar objetivos e metas, tem liberdade para tomada de decisões, 
identifica e gerencia os recursos. O intraempreendedor pode eleger suas estratégias de ação e tem a liberdade de elaborar planos de ação, para que assim, a organização atinja seus resultados, os quais podem impactar positivamente no desempenho organizacional (CAMELOORDAZ et al., 2012).

Estudos formam realizados com o efeito da entrada agressiva de organizações que se arriscavam em novos mercados e demostraram que estratégias agressivas impactam em maior desempenho organizacional, especialmente quanto à obtenção de maior retorno no investimento e crescimento do seu market share. As práticas de agressividade competitiva frequentemente relatadas pelos pesquisadores foram: definição de metas ambiciosas de market share, investimento na capacidade da nova infra-estrutura industrial, desenvolvimento e expansão das vendas, promoção de vendas, publicidade, qualidade e programas de preços significativos agressivos em relação aos concorrentes (LUMPKIN; DESS, 2001; AL-DHAAFRI; AL-SWIDI; YUSOFF, 2016). A agressividade competitiva conduz organizações a aproveitar as oportunidades em uma economia de transição, estando cada vez mais orientadas para o mercado, o que impacta significativamente no desempenho organizacional (ZHAO et al., 2011).

Além disso, os empreendedores podem ser observados como atores sociais, os quais usufruem dos benefícios advindos dos seus relacionamentos. Supostamente, o sistema de relações é o elemento fundamental, que é visto como um suporte para a prosperidade de uma visão de negócio. O estabelecimento de relações impacta diretamente na qualidade da visão de negócio do empreendedor, isso contribui para uma maior assertividade de suas ações, e consequentemente no desempenho organizacional. Por outro lado, essas ações postula o estabelecimento de novas relações, o que instiga o estabelecimento de novas visões do negócio (WANG, 2008; BOSO; STORY; CADOGAN, 2013). A troca de ideias por meio de redes de contatos (MAHONEY, 1995), é primordial para que o empreendedor reduza o risco de insucesso. Com base na literatura apresentada, propõem-se as seguintes hipóteses de pesquisa:

H4: A inovatividade tem um impacto positivo e significativo no desempenho organizacional.

H5: A assunção de riscos tem um impacto positivo e significativo na inovatividade.

H6: A proatividade do empreendedor tem um impacto positivo e significativo na assunção de riscos.

H7: A autonomia tem um impacto positivo e significativo no desempenho organizacional.

H8: A agressividade competitiva tem um impacto significativo no desempenho organizacional.

H9: A rede de relações tem um impacto positivo e significativo no desempenho organizacional.

Com base no construto orientação empreendedora, juntamente com seus elementos: a inovatividade, a assunção de riscos, a autonomia, a agressividade competitiva e a rede de relações há impacto direto no desempenho organizacional. A orientação empreendedora foi observada como uma qualidade individual do empreendedor, indivíduo o qual constantemente desafia a incerteza do ambiente por meio da implementação de ações corporativas (MILLER; FRIESEN, 1982). 


\subsection{Construto capacidades dinâmicas e suas dimensões, a capacidade gerencial, a capacidade mercadológica e a capacidade ambidestra}

A partir da revisão da literatura identificou-se que os empreendedores com capacidade gerencial têm uma melhor compreensão da situação de sua organização. $E$ assim, pode integrar informações internas e externas para formar uma estimativa confiável do seu desempenho (DEMERJIAN; LEV; MCVAY, 2012). Capacidade gerencial é considerada como um relevante e significativo ativo intangível organização, tornando-se uma necessidade emergente em um ambiente dinamicamente descontínuo (TSENG; LEE, 2014), proporcionando para que haja uma gestão eficiente do sistema de informações (PRASAD; GREEN, 2015). Também é considerada a capacidade que melhorara a eficiência organizacional, o compartilhamento do conhecimento e a aprendizagem organizacional, o que impacta positivamente no desempenho da organização (HUNG et al., 2010).

A capacidade mercadológica a partir da capacidade gerencial, isto é, a partir do conhecimento do empreendedor sobre a concorrência, clientes, suas habilidades e capacidades em segmentação de mercado impacta diretamente no desempenho superior das organizações (SONG, NASON; BENEDETTO, 2008). Em concordância, Silveira-Martins (2012) complementa este pensamento dizendo que a capacidade mercadológica pode ser observada como uma competência organizacional interna. Quando há capacidade gerencial, por parte dos empreendedores, a capacidade mercadológica é eficaz no processo de formulação de estratégias, avaliando assim, as circunstâncias ambientais, que por sua vez têm impacto direto sobre o desempenho organizacional. As referidas capacidades dinâmicas são mais eficazes na implementação da mudança organizacional em altos graus de dinamismo ambiental, isto é, a capacidade gerencial impacta na capacidade mercadológica (JIAO et al., 2013).

Logo, as empresas que almejam ter ações ambidestras necessitam planejar e implementar tipos específicos de mecanismos de integração em distintos níveis hierárquicos (JANSEN; VAN DEN BOSCH; VOLBERDA, 2005), vinculando a diversidade organizacional e a visão compartilhada (NEDZINSKAS et al., 2013). Segundo Chen e Ling (2010) para melhorar o desempenho organizacional, os líderes necessitam utilizar sua capacidade de equilibrar distintas exigências. Os empreendedores devem buscar múltiplas alternativas em suas tomadas de decisões, analisando os prós e contras do resultado das decisões. Desta forma, a capacidade gerencial, por meio da percepção do empreendedor do cenário impacta significativamente na capacidade ambidestra (PAl; CHANG, 2013). Dependendo de como os empreendedores perceberem o cenário, haverá a possibilidade de desenvolver um processo ambidestro, ou optar por explorar ou explotar (LI; LIN, 2008; WANG; SENARATNE; RAFIQ, 2015).

Jansen, Van Den Bosch e Volberda (2005) afirmam que a manutenção da ambidestria passa pelo contínuo ajuste das distintas variáveis que interferem no processo organizacional, tais como ambiente e o comportamento do empreendedor. Os autores idenficaram que em ambientes dinâmicos, a exploração é mais eficaz; e a explotação é em ambientes mais competitivos. Além do ambiente externo, o interno também tem significativa influência sobre a ambidestria organizacional. Han e Celly (2008) observam que novos empreendimentos que adotaram a ambidestria como estratégia desfrutam de um desempenho superior àqueles que não o fizeram. O fato de ser um novo empreendimento $e$, por consequência, haver determinadas restrições de recursos não os faz desistir da ambidestria como estratégia. Sendo assim, a capacidade ambidestra impacta no desempenho organizacional.

As capacidades mercadológicas podem produzir conhecimento de mercado superior, que por sua vez foi identificado como um recurso chave atrelado à capacidade das empresas 
de responder ao mercado e assegurar o crescimento do lucro, impactando na performance. As capacidades mercadológicas também apoiam o desempenho da empresa por responder às necessidades dos clientes de forma proativa (CHENG; CHEN; HUANG, 2014). Os empreendedores devem perceber que os efeitos das capacidades mercadológicas aumentam o uso das capacidades existentes de uma organização, a fim de aumentar o desempenho de seus novos produtos (CHEN et al., 2016). Assim, propõem-se as seguintes hipóteses de pesquisa:

H10: A capacidade gerencial tem um impacto positivo e significativo no desempenho organizacional.

H11: A capacidade gerencial tem um impacto positivo e significativo na capacidade mercadológica.

H12: A capacidade gerencial tem um impacto positivo e significativo na capacidade ambidestra.

H13: A capacidade ambidestra tem um impacto positivo e significativo no desempenho organizacional.

H14: A capacidade mercadológica tem um impacto positivo e significativo no desempenho organizacional.

As capacidades dinâmicas permitem ao empreendedor tomar decisões oportunas e orientadas para o mercado, inclusive, introduzir mudanças inovadoras na base de recursos, o que proporciona uma melhor performance organizacional (FAINSHMIDT et al., 2016). Assim, as capacidades abordadas por este estudo são: capacidade gerencial, capacidade ambidestra e a capacidade mercadológica, as quais estão diretamente relacionadas com o desempenho organizacional, ou seja, quanto são instigadas e trabalhadas, a organização pode alcançar o sucesso nos negócios.

\subsection{O papel moderador do tempo de mercado e o desenvolvimento de novos produtos}

A primeira variável moderadora, onde a literatura aponta que tempo de atuação no mercado modera a relação entre as variáveis independentes (capacidade gerencial e capacidade ambidestra) e a variável dependente (desempenho organizacional), sendo que tal relação será mais forte para empresas que atuam no mercado há mais tempo.

O tempo de atuação no mercado modera a relação entre a capacidade gerencial e o desempenho organizacional. Assim, a premissa de que a capacidade gerencial melhora o desempenho é uma afirmação empírica, contudo, estudos envolvendo dimensões de longo prazo que estão explorando este relacionamento são incomuns. Um estudo investigou o impacto do tempo de atuação da capacidade gerencial no desempenho organizacional, utilizando um período de 15 anos de avaliadores de propriedades locais, no Estado de Washington de 1999 a 2013. Assim, verificou-se que cada ano adicional de experiência melhora a qualidade de avaliação. Sendo possível observar que a relação foi estatisticamente positiva e significativa, instigando estudos futuros (PROPHETER, 2016).

O tempo de atuação no mercado modera a relação entre a capacidade ambidestra e o desempenho organizacional. March (1991) destaca a capacidade ambidestra envolvendo dois estágios, a exploração que está relacionada com a criação, inovação, invenção de produtos e serviços, o segundo estágio é a explotação, que está relacionada com ações de melhoria do que já foi desenvolvido no primeiro estágio. Com o tempo de atuação no mercado, as experiências diárias do empreendedor são ampliadas, permitindo assim que ele utilize os dois estágios de forma contínua, conquistando um movimento ambidestro. Para Lynn Jr. (1996), 
um líder reúne seus conhecimentos por meio da educação formal e ao longo de experiência empresarial.

Em concordância, McDowell, Harris e Geho (2015) existe uma relação positiva e significava entre o tempo de atuação da empresa no mercado e desempenho organizacional. Os resultados apontam que empresas mais jovens, em consonância com a responsabilidade, mostram um foco estratégico externo. Por outro lado, as empresas atuam há mais tempo no mercado são consistentes com a mudança organizacional e responsabilidade do amadurecimento, utilizando um foco estratégico interno. Com base na literatura, pretendese verificar se o tempo de atuação no mercado modera a relação entre o desempenho organizacional e as dimensões investigadas.

Por fim, a segunda variável moderadora refere-se que o nível de desenvolvimento de novos produtos modera a relação entre as variáveis independentes (dimensões inovatividade e capacidade mercadológica) e a variável dependente (desempenho organizacional), sendo que tal relação será mais forte para empresas que lançam mais produtos junto ao mercado.

O desenvolvimento de novos produtos modera a relação entre a inovatividade e o desempenho organizacional. Uma vez que, as organizações que possuem um comportamento inovador tendem a desenvolvem novos produtos. O objetivo da organização é aumentar seu portfolio de produtos, aumentar a lucratividade e atuar em outros mercados, o que impacta positivamente no desempenho organizacional. Uma melhor performance de um novo produto no mercado pode ser alcançada por meio da adoção de um conjunto de estratégia e ações que sirvam de suporte para a gestão de novos projetos. (URHAHN; SPIETH, 2014).

O desenvolvimento de novos produtos modera a relação entre a capacidade mercadológica e o desempenho organizacional. Sendo assim, há uma tendência das organizações em incluir o conhecimento de mercado, clientes ou concorrentes como forma de melhorar o desempenho de novos produtos. No entendimento de Li e Calantone (1998), essa tendência vem sendo observada nas práticas empresariais, a qual ocorre de forma simultânea ao desenvolvimento da literatura de marketing, cujo o foco é o conhecimento da competência do mercado. Neste sentido, alguns trabalhos podem ser destacados, como a relação entre desempenho organizacional e orientação para o mercado realizada por Narver e Slater (1990) e os avanços dos efeitos moderadores (KOHLI; JAWORSKI, 1993).

Portanto, a busca por melhor desempenho organizacional com o lançamento novos produtos têm se mostrado como algo imprescindível para empresas que tenham a finalidade de obter uma vantagem competitiva. Sendo assim, para obter uma vantagem competitiva e ter desempenho superior, uma organização deve continuamente gerar, disseminar e aplicar novos conhecimentos no desenvolvimento de novos produtos, isto é, empresas que lançam mais produtos junto ao mercado, apresentam uma performance superior. A mensuração de desempenho organizacional influencia a escolha dos indicadores (DE TONI; MILAN; REGINATO, 2011). Para Bonner (2010, p. 485), "o que está faltando a partir da literatura é uma conceituação mais refinada das dimensões do processo de interação com o cliente, e um exame de como essas dimensões influenciam o desempenho do desenvolvimento de novos produtos". Assim, apresenta-se as últimas hipóteses de pesquisa:

H15: O tempo de atuação da empresa no mercado modera a relação entre desempenho organizacional e: (a) capacidade gerencial e (b) capacidade ambidestra.

H16: O desenvolvimento de novos produtos modera a relação entre desempenho organizacional e: (a) inovatividade e (b) capacidade mercadológica.

Os propósitos centrais da mensuração do desempenho estão na melhoria da performance do processo interno e no aumento da performance organizacional, comparado 
à concorrência (SCHUMANN JUNIOR; RANSLEY, 1995). Assim, este estudo propõe no seu modelo conceitual a verificação de moderadores, como: tempo de atuação da empresa no mercado com capacidade gerencial e capacidade ambidestra e o desenvolvimento de novos produtos com inovatividade e capacidade mercadológica. Sugere-se verificar se estes têm relação com o desempenho organizacional e o quanto esta moderação é significativa.

\section{CONSIDERAÇÕES FINAIS}

Os recursos organizacionais compreendem os recursos tangíveis e intangíveis, que permitem a empresa delinear seus objetivos e implementar suas estratégias (OLAVARRIETA; ELLINGER, 1997). Os métodos de aquisição de recursos podem, portanto, ser mutuamente exclusivos, os quais poderão ser difíceis de ser imitados, para assim assegurar um desempenho superior. Os resultados alcançados nas pesquisas de Cai, Hughes e Yin (2014), contribuem para o conhecimento, uma vez destacam a relevância de o empreendedor estar disposto a enfrentar o "desafio empresarial". Desafio o qual, que conduzirá o empreendedor construir uma base de recursos, internos e externos, combinando distintos métodos para atingir o desempenho organizacional (LI; TAN, 2013).

Para Edvinsson e Malone (1997), os ativos e recursos intangíveis podem ser agrupados em relacionamento com clientes, conjunto de processos produtivos e de prestação de serviços, capacidade de renovação e desenvolvimento (R\&D). No que tange ao relacionamento com os clientes, entende-se a qualidade deste relacionamento, destacada como a capacidade da organização se antecipar na previsão das necessidades de seus clientes, e assim, direcionar sua demanda. O segundo grupo de ativos e recursos intangíveis está destacado como um conjunto de processos produtivos e de prestação de serviços da empresa. O terceiro grupo de ativos e recursos intangíveis está vinculado a capacidade de renovação e desenvolvimento da organização, representada pelas adaptações, às oportunidades futuras, ou motivadas, sem aviso prévio, por alterações no comportamento do mercado.

A relação entre orientação empreendedora e desempenho organizacional foi examinado por meio de diferentes tipos de pesquisa, como a abordagem meta-análise, empírica, conceitual, ou revisão de literatura. Os resultados alcançados por algumas dessas pesquisas, especificamente estudos empíricos, foram positivos e significativos (ZHANG; ZHANG, 2012; DADA; WATSON, 2013; ABEBE, 2014). Por outro lado, estudos não encontraram efeitos significativos entre OE e desempenho organizacional, devido a estes resultados inconsistentes, Al-Dhaafri e Al-Swidi (2016) indicam que são necessários mais estudos sobre a relação entre orientação empreendedora e desempenho organizacional.

Barreto (2010, p. 227) contribui afirmando que as capacidades dinâmicas podem solucionar sistematicamente os problemas, permitindo assim que o empreendedor "tome decisões orientadas para o mercado" e adentre mudanças inovadoras na base de recursos. Para a empresa desenvolver capacidades dinâmicas inicialmente é indispensável ter um conjunto de comportamentos e habilidades relacionadas à mudança e inovação. A estrutura das capacidades dinâmicas considera o empreendedor com o principal ator em uma organização, sendo responsável por reconhecer a necessidade de mudanças nos ambientes de negócios, onde há uma profunda incerteza (DRIESCH et al., 2015; TEECE, 2016).

Dentre as contribuições deste trabalho destaca-se, a proposição de diferentes dimensões que podem impactar no desempenho organizacional. Muitas podem ser as variáveis que impactam no desempenho das organizações, assim, a partir da revisão da literatura identificou-se que a base teórica baseada na Gestão de Recursos, na Orientação Empreendedora e nas Capacidades dinâmicas constituem relevantes elementos 
direcionadores que podem explicar a diferença no desempenho das organizações. Assim, este ensaio teórico contribui também para dar um direcionamento a futuras pesquisas mensurar o desempenho das organizações. Mostrando ainda que o desempenho organizacional é complexo demais para ser mensurado por uma ou duas variáveis apenas. Como uma variável dependente complexa, o desempenho organizacional, tanto do ponto de vista financeiro ou não, precisa ser tratado não apenas como um comportamento lógico, mas também como um resultado sistêmico em que fatores internos e externos se relacionam e impactam de diferentes formas nos resultados das organizações.

Portanto, sugere-se que o modelo teórico proposto por este estudo seja testado em um estudo quantitativo, em um determinado setor. O que contribuirá de forma gerencial, identificando quais são os fatores que conduzem as empresas ao seu desempenho, permitindo que gestores tenham mais comprovações empíricas para delinear suas estratégias. Este modelo também traz suas contribuições teóricas, novos estudos e seus elementos estratégicos ao tema desempenho organizacional.

\section{REFERÊNCIAS}

ABEBE, M. Electronic commerce adoption, entrepreneurial orientation and small and medium-sized enterprise (SME) performance. Journal of Small Business and Enterprise Development, v. 21, n. 1, p. 100-116, 2014.

ADAMS, J. H.; KHOJA, F. M.; KAUFFMAN, R. An empirical study of buyer-supplier relationships within small business organizations. Journal of Small Business Management, $v$. 50, n. 1, p. 20-40, 2012.

AL-DHAAFRI, H. S.; AL-SWIDI, A. The impact of total quality management and entrepreneurial orientation on organizational performance. International Journal of Quality \& Reliability Management, v. 33, n. 5, p. 597-614, 2016.

AL-DHAAFRI, H. S.; AL-SWIDI, A. K.; YUSOFF, R. Z. B. The mediating role of total quality management between the entrepreneurial orientation and the organizational performance. TQM Journal, v. 28, n. 1, p. 89-111, 2016.

ALLEE, V. Value network analysis and value conversion of tangible and intangible assets. Journal of Intellectual Capital, v. 9, n. 1, p. 5-24, 2008.

ANDREWS, R.; BOYNE, G. A. Capacity, leadership, and organizational performance: testing the black box model of public management. Public Administration Review, v. 70, n. 3, p. 443-454, 2010.

ARCAND, M.; ARCAND, G.; BAYAD, M.; FABI, B. Human resource management bundles and organizational performance: The case of the Canadian financial cooperative industry. Annals of Public and Cooperative Economics, v. 75, n. 3, p. 497-524, 2004.

ARORA, A.; ARORA, A. S.; SIVAKUMAR, K. Relationships among supply chain strategies, organizational performance, and technological and market turbulences. International Journal of Logistics Management, v. 27, n. 1, p. 206-232, 2016.

BARNEY, J. B. Strategic factor markets: expectations, luck, and business strategy.

Management Science, v. 32, n. 10, p. 1231-1241, 1986. 
BARRETO, I. Dynamic capabilities: a review of past research and an agenda for the future. Journal of Management, v. 36, n. 1, p. 256-280, 2010.

BONNER, J. M. Customer interactivity and new product performance: Moderating effects of product newness and product embeddedness. Industrial Marketing Management, v. 39, p. 485-492, 2010.

BONTIS, N. National intellectual capital index: a united nations initiative for the arab region. Management Decision, v. 36, n. 2, p. 63-76, 1998.

BOSO, N.; STORY, V. M.; CADOGAN, J. W. Entrepreneurial orientation, market orientation, network ties, and performance: Study of entrepreneurial firms in a developing economy. Journal of Business Venturing, v. 28, n. 6, p. 708-727, 2013.

BOULTON, R. S.; LIBERT, B. D.; SAMEK, S. M. Cracking the value code. New York: Harper Collings Publishers, 2001.

CAMELO-ORDAZ, C.; FERNÁNDEZ-ALLES, M.; RUIZ-NAVARRO, J.; SOUSA-GINEL, E. The intrapreneur and innovation in creative firms. International Small Business Journal, v. 30, n. 5, p. 513-535, 2012.

CARTON, R. B.; HOFER, C. W. Measuring organizational performance: metrics for entrepreneurship and strategic management research. Cheltenham, UK; Northampton, MA: Edward Elgar, 2006.

CHEN, K. H.; WANG, C. H.; HUANG, S. Z.; SHEN, G. C. Service innovation and new product performance: The influence of market-linking capabilities and market turbulence. International Journal of Production Economics, v. 172, p. 54-64, 2016.

CHENG, J. H.; CHEN, M. C.; HUANG, C. M. Assessing inter-organizational innovation performance through relational governance and dynamic capabilities in supply chains. Supply Chain Management, v. 19, n. 2, p. 173-186, 2014.

CHEN, Y. C.; LI, P. C.; EVANS, K. R. Effects of interaction and entrepreneurial orientation on organizational performance: Insights into market driven and market driving. Industrial Marketing Management, v. 41, p. 1019-1034, 2012.

COLLINS, C. J.; CLARK, K. D. Strategic human resource practices, top management team social networks, and firm performance: the role of human resource practices in creating organizational competitive advantage. Academy of Management Journal, v. 46, n. 6, p. 740752, 2003.

COVIN, J. G.; MILES, M. P. Corporate entrepreneurship and the pursuit of competitive advantage. Entrepreneurship: Theory \& Practice, v. 23, n. 3, p. 47-63, 1999.

DADA, O.; WATSON, A. Entrepreneurial orientation and the franchise system organizational antecedents and performance outcomes. European Journal of Marketing, v. 47, n. 5/6, p. 790-812, 2013.

DEMERJIAN, P.; LEV, B.; MCVAY, S. Quantifying managerial ability: A new measure and validity tests. Management Science, v. 58, n. 7, p. 1229-1248, 2012. 
DE TONI, D.; MILAN, G. S.; REGINATO, C. E. R. Fatores críticos para o sucesso no desempenho de novos produtos: um estudo aplicado ao setor moveleiro da Serra Gaúcha. Revista Gestão \& Produção, v. 18, n. 3, p. 587-602, 2011.

DRIESCH, T. V. D.; COSTA, M. E. S.; FLATTEN, T. C.; BRETTEL, M. How CEO experience, personality, and network affect firms' dynamic capabilities. European Management Journal, v. 33, n. 4, p. 245-256, 2015.

EDVINSSON, L.; MALONE, M.S. Intellectual capital: realizing your company's true value by finding its hidden brainpower. Harper Business: New York, 1997.

ENGELEN, A.; GUPTA, V.; STRENGER, L.; BRETTEL, M. Entrepreneurial orientation, firm performance, and the moderating role of transformational leadership behaviors. Journal of Management, v. 41, n. 4, p. 1069-1097, 2015.

FAINSHMIDT, S.; PEZESHKAN, A.; LANCE FRAZIER, M.; NAIR, A.; MARKOWSKI, E. Dynamic capabilities and organizational performance: a meta-analytic evaluation and extension. Journal of Management Studies, v. 53, n. 8, p. 1348-1380, 2016.

FELÍCIO, J. A.; COUTO, E.; CAIADO, J. Human capital, social capital and organizational performance. Management Decision, v. 52, n. 2, p. 350-364, 2014.

FERNÁNDEZ-MESA, A.; ALEGRE-VIDAL, J.; CHIVA-GÓMEZ, R. Entrepreneurial orientation, organizational learning capacity and innovative performance. Journal of Technology Management and Innovation, v. 7, n. 2, p. 157-170, 2012.

GARENGO, P.; BIAZZO, S.; BITITCI, U. S. Performance measurement systems in SMEs: A review for a research agenda. International Journal of Management Reviews, v. 7, n. 1, p. 25-47, 2005.

GARENGO, P.; NUDURUPATI, S.; BITITCI, U. Understanding the relationship between PMS and MIS in SMEs: an organizational life cycle perspective. Computers in Industry, v. 58, n. 7, p. 677-686, 2007.

GRANDE, J.; MADSEN, E. L.; BORCH, O. J. The relationship between resources, entrepreneurial orientation and performance in farm-based ventures. Entrepreneurship \& Regional Development, v. 23, n. 3/4, p. 89-111, 2011.

GRANT, R. M. The Resource-based theory of competitive advantage: implications for strategy formation. California Management Review, v. 33, n.3, p.114-135, 1991.

GREEN, K.W.; INMAN, R. Using a just-in-time selling strategy to strengthen supply chain linkages. International Journal of Production Research, v. 43, n. 16, p. 3437-3453, 2005.

GUO, H.; TANG, J.; SU, Z. To be different, or to be the same? The interactive effect of organizational regulatory legitimacy and entrepreneurial orientation on new venture performance. Asia Pacific Journal of Management, v. 31, n. 3, p. 665-685, 2014.

HAKONSSON, D. D. How misfits between managerial cognitive orientations and situational uncertainty affect organizational performance. Simulation Modelling Practice and Theory, v. 14, n. 4, p. 385-406, 2006.

HAN, M.; CELLY, N. Strategic ambidexterity and performance in international new ventures. 
Canadian Journal of Administrative Science, v. 25, n. 4, p. 335- 349, 2008.

HENDERSON R. M. Managing innovation in the information age. Harvard Business Review, v. 72, n.1, p.100-106, 1994.

$\mathrm{HU}, \mathrm{Y}$. Role of organizational learning in the relationship between entrepreneurial orientation and performance of nonprofit organizations. Journal of Applied Sciences, v. 13, n. 14, p. 2795-2800, 2013.

HULT, G. T. M.; HURLEY, R. F.; KNIGHT, G. A. Innovativeness: Its antecedents and impact on business performance. Industrial Marketing Management, v. 33, p. 429-438, 2004.

HUNG, R. Y. Y.; YANG, B.; LIEN, B. Y. H.; MCLEAN, G. N.; KUO, Y. M. Dynamic capability: Impact of process alignment and organizational learning culture on performance. Journal of World Business, v. 45, n. 3, p. 285-294, 2010.

JANSEN, J.; VAN DEN BOSCH, V.; VOLBERBA, H. Managing potential and realized absorptive capacity: how do organizational antecedents matter? Academy Management Journal, v. 48, n. 6, p. 999-1015, 2005.

JAWORSKI, B. J.; KHOLI, A. K. Market orientation: antecedents and consequences. Journal of Marketing, v. 57, n. 3, p.53-70, 1993.

JIAO, H.; ALON, I.; KOO, C.K.; CUI, Y. When should organizational change be implemented? the moderating effect of environmental dynamism between dynamic capabilities and new venture performance. Journal of Engineering and Technology Management, v. 30, n. 2, p. 188-205, 2013.

LI, T.; CALANTONE, R. J. The impact of marketing knowledge competence on new product advantage: Conceptualization and empirical examination. Journal of Marketing, v. 62, p. 1329, 1998.

KATOU, A. A. Investigating reverse causality between human resource management policies and organizational performance in small firms. Management Research Review, v. 35, n. 2, p. 134-156, 2012.

. The mediating effects of psychological contracts on the relationship between human resource management systems and organisational performance. International Journal of Manpower, v. 36, n. 7, p. 1012-1033, 2015.

LAUD, G.; KARPEN, I. O.; MULYE, R.; RAHMAN, K. The role of embeddedness for resource integration: complementing S-D logic research through a social capital perspective.

Marketing Theory, v. 15, n. 4, p. 509-543, 2015.

LI, C. R; LIN, C-J. The nature of market orientation and the ambidexterity of innovations. Management Decision, v. 46, n. 7, p. 1002-1026, 2008.

LI, S., SUBBA RAO, S., RAGU-NATHAN, B., RAGU-NATHAN, T. Development and validation of measurement instruments for studying supply chain management practices. Journal of Operations Management, v. 23, n. 6, p. 618-641, 2005.

LI, Y.; TAN, C. H. Matching business strategy and $\mathrm{ClO}$ characteristics: The impact on organizational performance. Journal of Business Research, v. 66, n. 2, p. 248-259, 2013. 
LIN, C. H. V.; SANDERS, K.; SUN, J. M. J.; SHIPTON, H.; MOOI, E. A. From Customer-Oriented Strategy to Organizational Financial Performance: The Role of Human Resource Management and Customer-Linking Capability. British Journal of Management, 2016.

LU, C. M.; CHEN, S. J.; HUANG, P. C.; CHIEN, J. C. Effect of diversity on human resource management and organizational performance. Journal of Business Research, v. 68, n. 4, p. 857-861, 2015.

LUMPKIN, G. T.; DESS, G. Clarifying the entrepreneurial orientation construct and linking it to performance. Academy of Management Review, v. 21, n. 1, p. 135-172, 1996.

Linking two dimensions of entrepreneurial orientation to firm performance: the moderating role of environment and industry life cycle. Journal of Business Venturing, v. 16, n. 5, p. 429-451, 2001.

MAHONEY, J. The management of resources and the resource of management. Journal of Business Research, v. 33, n. 2, p. 91-101, 1995.

$\mathrm{MARCH}$, J. G. Exploration and exploitation in organization learning. Organization Science, v.2, n.1, p. 71-87, 1991.

MARTINHO, J. L.; GOMES, C. F.; YASIN, M. M. The role of people and social context in promoting the IT organizational performance: evidence from Portugal. Personnel Review, $\mathrm{v}$. 45, n. 5, p. 1087-1107, 2016.

MARTINS, R. A. Sistemas de medição de desempenho: um modelo para estruturação do uso. 1999. 269 f. Tese (Doutorado em Engenharia) - Escola Politécnica da Universidade de São Paulo, Programa de Doutorado em Engenharia, 1999.

McDOWELL, W.; HARRIS, M. L; GEHO, P. R. Longevity in small business: The effect of maturity on strategic focus and business performance. Journal of Business Research, p. 1-5, 2015.

MELLO, S. C. B.; LEÃO, A. L. M. S. Compreendendo a orientação empreendedora de empresas de alta tecnologia. In: SOUZA, E. C. L.; GUIMARÃES, T. A. (Orgs.),

Empreendedorismo além do plano de negócios. São Paulo: Atlas, 2005, p. 162-178.

MILLER, D. The correlates of entrepreneurship in three types of firms. Management Science, v. 29, n.7, 770-791, 1983.

MILLER, D.; FRIESEN, P.H. Innovation in conservative and entrepreneurial firms: two models of strategic momentum. Strategic Management Journal, v. 3, n.1, p. 1-26, 1982.

MORRIS, M. H.; ALLEN, J.; SCHINDEHUTTE, M.; AVILA, R. Balanced management control systems as a mechanism for achieving corporate entrepreneurship. Journal of Management, v. 17, n. 4, p. 468-493, 2006.

NARVER, J. C.; SLATER, S. F. The Effect of market orientation on business profitability. Journal of Marketing, v. 54, n. 4, p. 20-35, Oct. 1990.

NEDZINSKAS, S.; PUNDZIENE, A.; BUOZIUTE-RAFANAVICIENE; S., PILKIENE, M. The impact of dynamic capabilities on SME performance in a volatile environment as moderated by organizational inertia. Baltic Journal of Management, v. 8, n. 4, p. 376-396, 2013. 
OGUNYOMI, P.; BRUNING, N. S. Human resource management and organizational performance of small and medium enterprises (SMEs) in Nigeria. International Journal of Human Resource Management, v. 27, n. 6, p. 612-634, 2016.

OLAVARRIETA, S.; ELLINGER, A. E. Resource-based theory and strategic logistics research. International Journal of Physical Distribution \& Logistics Management, v. 27, n. 9-10, p. 559-587, 1997.

PAI, F. Y.; CHANG, H. F. The effects of knowledge sharing and absorption on organizational innovation performance - A dynamic capabilities perspective. Interdisciplinary Journal of Information, Knowledge, and Management, v. 8, p. 83-97, 2013.

PARVEEN, F.; JAAFAR, N. I.; AININ, S. Social media's impact on organizational performance and entrepreneurial orientation in organizations. Management Decision, v. 54, n. 9, p. 22082234, 2016.

PRASAD, A.; GREEN, P. Organizational competencies and dynamic accounting information system capability: Impact on ais processes and firm performance. Journal of Information Systems, v. 29, n. 3, p. 123-149, 2015.

PROPHETER, G. Managerial experience and organizational performance: A 15-Year Panel Study of Local Assessors. Public Administration Review, v. 76, n. 3, p. 438-446, 2016.

RAUCH, A.; WIKLUND, J.; LUMPKIN, G. T.; FRESE, M. Entrepreneurial orientation and business performance: an assessment of past research and suggestions for the future. Entrepreneurship Theory and Practice, v. 34, n. 5, 761-787, 2009.

REILLY, R. F.; SCHWEIHS, R. P. Valuing intangible assets. NY: McGraw-Hill, 1998.

SANDBERG, B. Creating the market for disruptive innovation: market proactiveness at the launch stage. Journal of Targeting, Measurement and Analysis for Marketing, v. 11, n. 2, p. 184-196, 2002.

SCHUMANN JUNIOR, P.; RANSLEY, D. L. Measuring R\&D performance. Research Technology Management, v. 38, n. 3, p. 45-54, 1995.

SCOTT, W. R. The organization of environments: network, cultural, and historical elements. In: MEYER, J. W.; SCOTT, W. R. (Eds.). Organizational environments: ritual and rationality. ed. rev. atual. London: Sage Publications, 1992.

SHEEHAN, N. T. Understanding how resources and capabilities affect performance: Actively applying the resource-based view in the classroom. Journal of Management Education, v. 30, n. 3, p. 421-430, 2006.

SILVEIRA-MARTINS, E. Comportamento estratégico, ambidestria, incerteza ambiental e desempenho no processo de formulação de estratégias de empresas vinícolas 16 brasileiras. 2012. $201 \mathrm{fls}$. Tese (Doutorado em Administração) - Universidade do Vale do Itajaí, Programa de Doutorado em Administração, 2012.

SONG, M.; NASON, R. W.; BENEDETTO, C. A. D. Distinctive marketing and information technology capabilities and strategic types: a cross-national investigation. Journal of International Marketing. v. 16, n. 1, p. 4-38, 2008. 
SOUSA, S. D.; ASPINWALL, E. M.; RODRIGUES, A. G. Performance measures in English small and medium enterprises: Survey results. Benchmarking, v. 13, n. 1/2, p. 120-134, 2006.

SRIVASTAVA, A. P.; DHAR, R. L. Impact of leader member exchange, human resource management practices and psychological empowerment on extra role performances: The mediating role of organisational commitment. International Journal of Productivity and Performance Management, v. 65, n. 3, p. 351-377, 2016.

SUN, L. Y.; PAN, W. Market orientation, intrapreneurship behavior, and organizational performance: Test of a structural contingency model. Journal of Leadership and Organizational Studies, v. 18, n. 2, p. 274-285, 2011.

TEECE, D. J. Dynamic capabilities and entrepreneurial management in large organizations: Toward a theory of the (entrepreneurial) firm. European Economic Review, v. 86, p. 202216, 2016.

TEECE, D. J.; PISANO, G.; SHUEN, A. Dynamic capabilities and strategic management. In: FOSS, N. J. (Ed.). Resources firms and strategies - a reader in the resource-based perspective. Oxford: Oxford University Press, p. 268-285, 1997.

TSENG, C. H.; LEE, R. P. The effect of knowledge management capability and dynamic capability on organizational performance. Journal of Enterprise Information Management, v. 27, n. 2, p.158-179, 2014.

URHAHN, C.; SPIETH, P. Governing the portfolio management process for product innovation-a quantitative analysis on the relationship between portfolio management governance, portfolio innovativeness, and firm performance. IEEE Transactions on Engineering Management, v. 61, n. 3, 2014.

ZHANG, Y.; ZHANG, X. The effect of entrepreneurial orientation on business performance a role of network capabilities in China. Journal of Chinese Entrepreneurship, v. 4, n. 2, p. 132142, 2012.

ZHAO, P.; YUAN, Y.; GUO, Y. Brief analysis on human resources management in modern private enterprises. Asian Social Science, v. 7, n. 3, p. 232-236, 2011.

WANG, C. L. Entrepreneurial orientation, learning orientation, and firm performance. Entrepreneurship Theory and Practice, v. 32, n. 4, p. 635-657, 2008.

WANG, C. L.; SENARATNE, C.; RAFIQ, M. Success traps, dynamic capabilities and firm performance. British Journal of Management, v. 26, n. 1, p. 26-44, 2015.

WEILL, P.; VITALE, M. What IT infrastructure capabilities are needed to implement e-business models. MIS quarterly Executive, v. 1, n. 1, p. 17-34, 2002.

WERNERFELT, B. A resource-based view of the firm. Strategic Management Journal, v. 5, n. 2, p. 171-180, 1984. 